\title{
Improved Limits on Variation of the Fine Structure Constant and Violation of Local Position Invariance
}

T.M. Fortier, N. Ashby, J.C. Bergquist, M.J. Delaney, S.A. Diddams, T.P. Heavner, L. Hollberg, W.M. Itano, S.R. Jefferts, K. Kim, W.H. Oskay, T.E. Parker, J.

Shirley, and J.E. Stalnaker

Time and Frequency Division MS 847

National Institute of Standards and Technology

Boulder, CO USA

Abstract - We report tests of Local Position Invariance (LPI) and constancy of fundamental constants from measurements of the frequency ratio of the $282-\mathrm{nm}^{199} \mathrm{Hg}^{+}$optical clock transition to the ground-state hyperfine splitting in ${ }^{133} \mathrm{Cs}$. Analysis of the frequency ratio, extending over six years at NIST, is used to place a limit on the fractional variation of the two clocks of less than $5.8 \times 10^{-6}$ per change in normalized solar gravitational potential, and a limit on fractional variation of the fine structure constant at $\frac{\dot{\alpha}}{\alpha}<1.3 \times 10^{-16} y r^{-1}$, assuming invariance of other fundamental constants. Comparison of our results with those previously reported for the absolute optical frequency measurements of ${ }^{171} \mathrm{Yb}^{+}$versus other ${ }^{133} \mathrm{Cs}$ standard yields a coupled constraint

of

$$
\begin{aligned}
& -0.04 \times 10^{-15}<\frac{\dot{\alpha}}{\alpha}<0.46 \times 10^{-15} y r^{-1} \text { and } \\
& -2.39 \times 10^{-15}<\frac{d}{d t} \ln \frac{\mu_{C s}}{\mu_{B}}<0.47 \times 10^{-15} y r^{-1} .
\end{aligned}
$$

\section{INTRODUCTION}

The comparisons of relative rates over time of atomic clocks based on different atoms provide some of the most sensitive measurements of the stability of fundamental constants and the possible violation of local position invariance. Our groups in the Time and Frequency Division of NIST now have over six years of measurements of the relative frequency of ${ }^{133} \mathrm{Cs}$ hyperfine transition (the definition of the SI second) against that of the $282 \mathrm{~nm}$ optical clock transition in ${ }^{199} \mathrm{Hg}^{+}$.

In the case of Cs, the hyperfine transition frequency is measured using NIST-F1, the U.S. primary frequency standard [1]. The ${ }^{199} \mathrm{Hg}^{+}$optical clock transition frequency is measured through excitation of a single ion confined in an RF trap using an ultrastable narrow linewidth laser system [2]. The

\author{
F. Levi and L. Lorini \\ Istituto Nazionale di Ricerca Metrologica \\ Strada delle Cacce 91 I-10135 \\ Torino, Italy
}

measurement uncertainties have decreased over this six year interval due to a variety of reasons. The statistical and systematic uncertainties in both the ${ }^{133} \mathrm{Cs}$ and ${ }^{199} \mathrm{Hg}^{+}$clocks have been reduced. Additionally, the optical frequency comb techniques [3], used to link the optical and microwave domains, have improved dramatically over the last several years.

The methods and data analysis used have been discussed in depth in a recent publication [4]. In these proceedings we review our main results and update other results using recent measurements that were unavailable at the time of our earlier publication.

\section{MEASUREMENTS AND ANALYSIS}

\section{A. Variation of Fundamental Constants}

The ratio of the two clock frequencies over time can be analyzed by several methods. The relationship between the variation in the ratio of the clock frequencies and the variation in $\alpha$ can be written as

$$
\frac{\frac{d}{d t}\left(\frac{{ }^{{ }_{H g^{+}}}}{v_{C s}}\right)}{\left(\frac{v_{H g^{+}}}{v_{C s}}\right)}=\frac{\dot{\alpha}}{\alpha} N-\frac{d}{d t} \ln \frac{\mu_{C s}}{\mu_{B}},
$$

where $\dot{\alpha}$ is the time rate of change in the fine structure constant, $N$ is a dimensionless constant that depends on the species of atoms used in ratio, $\mu_{C s}$ is the magnetic moment of Cs and $\mu_{B}$ is the Bohr magneton.

One way to analyze the data, shown in Fig.1, is to assume that the ratio $\mu_{C S} / \mu_{B}$ remains constant and that any

Work of the U.S. government. Not subject to copyright. 
variation is due to changes in $\alpha$. Based on this model our results give a value of

$$
\frac{\dot{\alpha}}{\alpha}=(-6.2 \pm 6.5) \times 10^{-17} y r^{-1} .
$$

This limit is a 20 -fold improvement over our previously reported limit [5].

Another model is to assume that any variation in the frequency ratio is due to a combination of variations in $\alpha$ and the ratio $\mu_{C s} / \mu_{B}$. In this case our data can be used with other measurements to place constraints on the variation of both quantities. Here we use recent results from [6] (not available at the time of our previous publication) to place new constraints. The new limits, shown in Fig.2, are defined by the area within the ellipse.

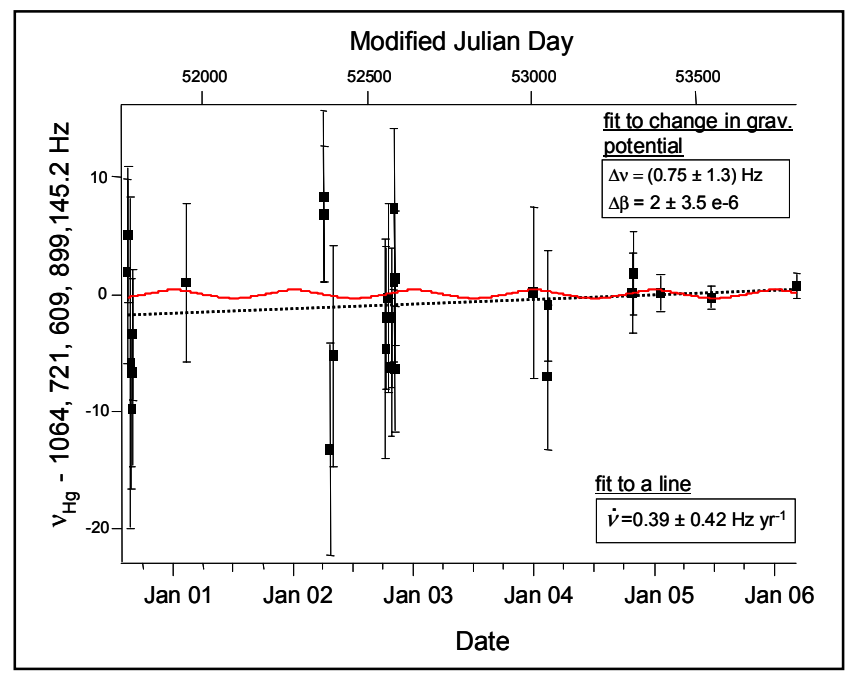

Figure 1. The history of the NIST ${ }^{199} \mathrm{Hg}^{+}$absolute frequency measurements against Cs (SI second) using NIST-F1. Shown are fits to both a linear drift and a solar gravitational potential used to extract limits on $\dot{\alpha}$ and LPI violation.

\section{B. Local Position Invariance}

Since the measurements occurred at random interval over the six-year span, we can look for a variation in the frequency ratio due to the solar potential that places limits on the violation of local position invariance. General relativity predicts the fractional frequency difference, $\Delta v / v$, between two identical clocks at different gravitational potentials, $\Delta \Phi=\Phi_{2}-\Phi_{1}$ to be

$$
\frac{\Delta v}{v}=\frac{\Delta \Phi}{c^{2}} .
$$

If local position invariance is violated, then the frequency shift will depend on the type of atomic clock. Therefore if two clocks of different internal structure, such as Cs and ${ }^{199} \mathrm{Hg}^{+}$, experience the same gravitational potential difference there will be a fractional frequency shift given by

$$
\frac{\Delta v}{v}=\left(\beta_{C s}-\beta_{H g^{+}}\right) \frac{\Delta \Phi}{c^{2}}
$$

where $\beta_{C s}$ and $\beta_{\mathrm{Hg}^{+}}$are dimensionless parameters describing the clocks' sensitivity to LPI violation.

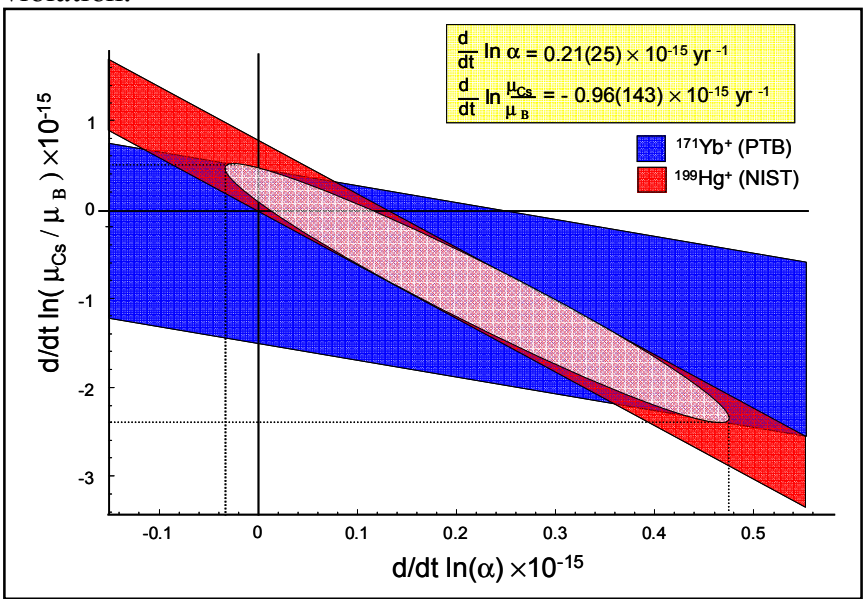

Figure 2. The constraints obtained on the variation of both $\alpha$ and

$\mu_{C s} / \mu_{B}$ obtained from our measurements in conjunction with the results of the frequency measurements of the ${ }^{171} \mathrm{Yb}^{+}$optical clock from Ref [6].

Our frequency measurements were fitted to a function describing the variation in the solar potential, and the result is

$$
\beta_{C S}-\beta_{H g^{+}}=(2 \pm 3.5) \times 10^{-6} \text {. }
$$

\section{SUMMARY}

Our result for the limit on the time variation on $\alpha$ is compared with results from astrophysical and geophysical measurements in [4]. Although these measurements place a constraint on $\left|\frac{\dot{\alpha}}{\alpha}\right| \simeq 10^{-16} \mathrm{yr}^{-1}$ and $10^{-17} \mathrm{yr}^{-1}$ respectively, there is disagreement over the models used to obtain these results.

The long-term comparison of atomic frequencies as shown here can also be used with other fundamental measurements and more elaborate models to place even tighter constraints on the variation of fundamental constants. A discussion of such models can be found in [7].

\section{REFERENCES}

[1] T.P. Heavner, S.R. Jefferts, E.A. Donley, J.H. Shirley, and T.E. Parker, "NIST-F1:Recent Results and Accuracy Evaluations," Metrologia, 42, 411-422, Sept. 2005.

[2] W.H. Oskay, S.A. Diddams, E.A. Donley, T. Fortier, T.P. Heavner, L. Hollberg, W.M. Itano, S.R. Jefferts, M.J. Jensen, K. Kim, F. Levi, T.E. Parker and J.C. Bergquist, "A Single-Ion Optical Clock with High Accuracy,” Phys. Rev. Lett., 97, 020801(4), July 2006.

[3] T.M. Fortier, A. Bartels and S.A. Diddams, Opt. Lett., 31, 1011, 2006. 
[4] T. Fortier, N. Ashby, J.C. Bergquist, M.J. Delaney, S.A. Diddams, T.P. Heavner, L. Holberg, W.M. Itano, S.R. Jefferts, K. Kim, F. Levi, L. Lorini, W.H. Oskay, T.E. Parker, J.H. Shirley, and J.E. Stalnaker, "Precision Atomic Specstroscopy for Improved Limits on Variation of the Fine Structure Constant and Local Position Invariance," Phys. Rev. Lett., 98, 070801(4), 2007.
[5] S. Bize et al., Phys. Rev. Lett. 90, 150802 (2003).

[6] E. Peik, B. Lipphardt, H. Schnatz, Chr. Tamm, S. Weyers, R. Wynands, "Laboratory Limits on Temporal Variations of Fundamental Constants:An Update," arXiv:physics/0611088v1, 9 Nov 2006.

[7] V.V. Flambaum, "Variation of Fundamental Constants," arXiv:0705.3704v1 [physics.atom-ph] 25 May 2007. 